\title{
REDUCTION OF THE PHOTOELECTRON YIELD FROM A COPPER BEAM CHAMBER BY SAW-TOOTH MACHINING
}

\author{
Y. Suetsugu, Y. Tanimoto, Y. Hori, K.Kanazawa and M. Kobayashi, KEK, Tsukuba, Japan \\ Y-J. Hsu, SRRC, Hsinchu, Taiwan, R.O.C.
}

\begin{abstract}
One of the latest serious problems for KEKB is a beam size blow up of the positron beam. The blow up is considered to be caused by a single-beam instability due to an electron cloud around the positron beam. The seed of the electrons is mainly photoelectrons emitted from the surface of vacuum chamber. Here a surface with a sawtooth structure is proposed to reduce the photoelectron yield. Experiments using a test chamber made of copper verify its validity.
\end{abstract}

\section{INTRODUCTION}

The KEK B-factory (KEKB) is an electron-positron collider with asymmetric energies to detect the $\mathrm{CP}$ violation in bottom-quark decay [1]. The KEKB consists of two rings, that is, the High Energy Ring (HER) for 8.0 $\mathrm{GeV}$ electrons and the Low Energy Ring (LER) for 3.5 $\mathrm{GeV}$ positrons. Most of beam chambers are made of OFC (oxygen free copper) for its ability to withstand an intense heat load and to effectively shield the radiation from the beam [2].

At present, the blow up of the vertical beam size of the positron beam is the most important issue for KEKB since it limits the improvement of the luminosity. Many simulations have been performed and the blow up is now considered to be due to the electron cloud around the beam. This cloud would excite a head-tail type singlebeam instability [3]. The seed of the electron cloud is mainly the photoelectrons emitted from the chamber surface irradiated by synchrotron radiation (SR). Some electrons may be multiplied by multipactoring.

An essential countermeasure against blow up is, therefore, to reduce the photoelectron yield from the chamber surface. A promising way is to machine the chamber surface like a saw-tooth, where the SR hits the surface at almost normal angle. This saw-tooth surface would reduce not only the photoelectron yield, but also the reflection of SR. An experiment performed at CERN for the LHC using SR with a critical energy of several ten $\mathrm{eV}$ showed an apparent reduction of the photoelectron yield [4]. For a higher energy photon, such as $6 \mathrm{keV}$ of the LER, however, the effect has not been verified.

The purpose of this study is to investigate the effect of a saw-tooth surface and its availability for the LER. We made a test chamber of copper with a saw-tooth surface and measured the photoelectron yield using the SR with a critical energy of $4.1 \mathrm{keV}$ from the KEK Photon Factory (PF). The spatial distribution of photoelectrons and the effect of the positive potential assuming a positron beam were also observed.

\section{EXPERIMENTAL SET UP}

The BL-21 beam line at PF at KEK was used for the study. The SR enters the beam line through a square slit $\left(5 \times 5 \mathrm{~mm}^{2}\right)$. The critical energy of SR is $4.1 \mathrm{keV}$. The total photon number is about $4.9 \times 10^{13}$ photons $\mathrm{s}^{-1}$ for a unit beam current in $\mathrm{mA}$.

The structure of a test chamber is shown in Figure 1. The test chamber is made of OFC and the diameter is 94 $\mathrm{mm}$, the same as the beam chamber for the LER. A 300 $\mathrm{mm}$ long test chamber is irradiated at a mean incidence angle of 3 degrees $(52 \mathrm{mrad})$. The irradiated length is about $100 \mathrm{~mm}$, i.e., from $50 \mathrm{~mm}$ to $150 \mathrm{~mm}$ from the front end of the test chamber. In order to see the spatial photoelectron distribution, 15 copper electrodes (12 $\mathrm{mm} \mathrm{x}$ $30 \mathrm{~mm}$ ), five rows axially (No.1- No.5) and three lines azimuthally $(\mathrm{A}, \mathrm{B}, \mathrm{C})$, are arranged above the irradiated area (see Figure 1). The axial and azimuthal spacing are $50 \mathrm{~mm}$ and 30 degrees, respectively. At the end of the chamber, an extra electrode of $50 \mathrm{~mm}$ in diameter, $\mathrm{D}$, is
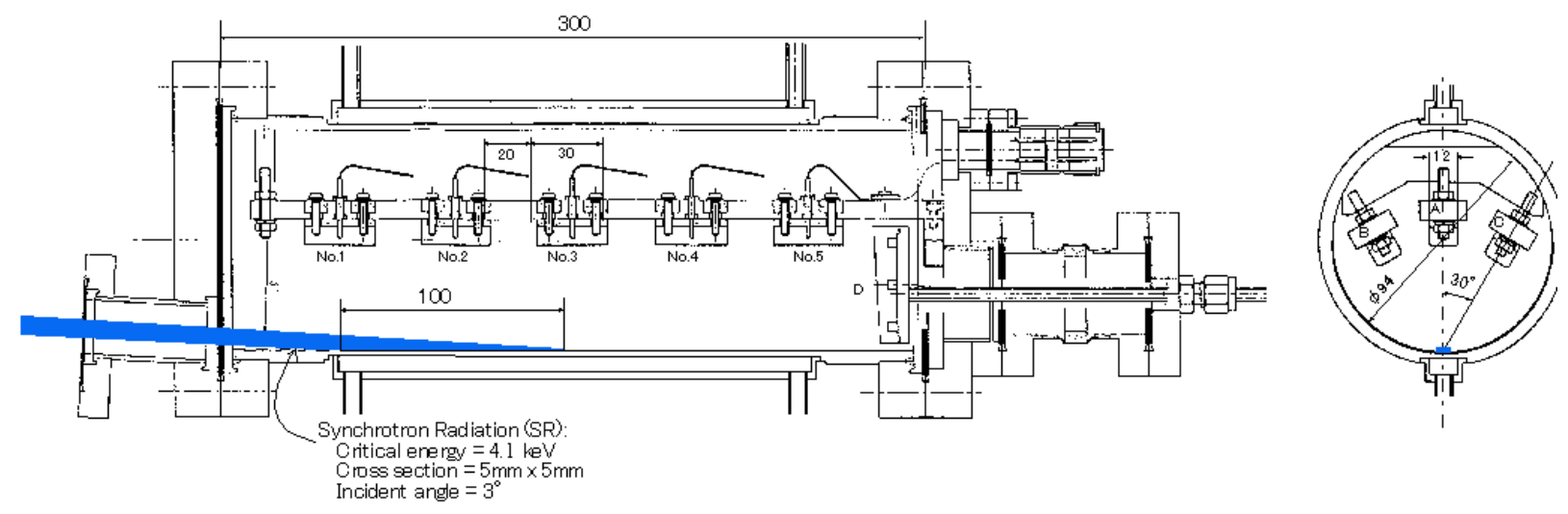

Figure 1: Structure of the test chamber and the electrodes inside it. 


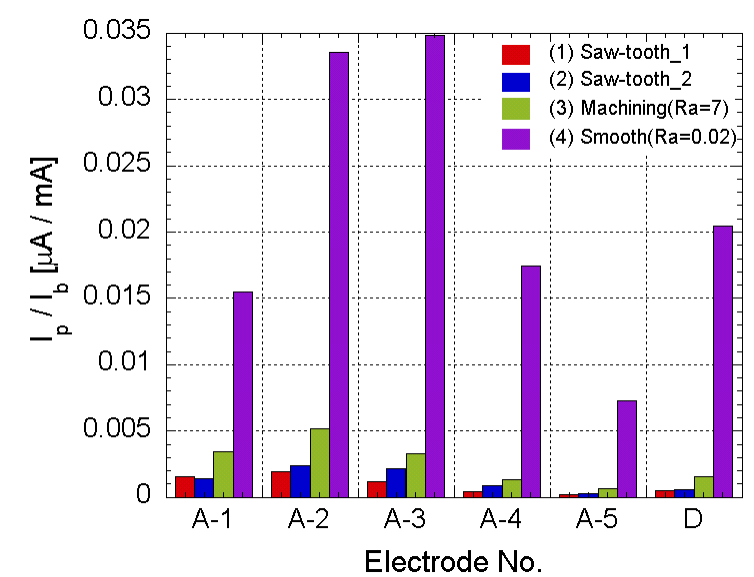

Figure 2: Photoelectron current $\left(I_{p}\right)$ normalized by the beam current $\left(I_{b}\right)$ for A and D electrodes.

set to estimate the reflectivity. A bias voltage can be applied to each electrode independently.

We measured the photoelectron yield for four different surfaces:

(1)[Saw-tooth_1] The saw-tooth surface with a pitch and a depth of $10 \mathrm{~mm}$ and $1 \mathrm{~mm}$, respectively. The machining was performed for a half of the chamber surface.

(2)[Saw-tooth_2] The saw-tooth surface is the same as (1), but machining was done only for about $20 \mathrm{~mm}$ width around the irradiated area.

(3) [Machining $(\mathrm{Ra}=7)$ ] The surface was lathed azimuthally with a mean roughness $(\mathrm{Ra})$ of about 7 .

(4) [Smooth $(\mathrm{Ra}=0.02)]$ A cold-drawn chamber same as that used for the LER.

For every case, the chamber was not baked. The surface was only degreased by an organic solvent. The total pressure in the test chamber during the irradiation was on the order of $10^{-4} \mathrm{~Pa}$.

\section{RESULTS AND DISCUSSIONS}

The following measurements were performed after the integrated photon irradiation of about $3 \times 10^{21}$ photons $\mathrm{m}^{-1}$, where the photoelectron yield settled down to almost a constant value.

\subsection{Energy of photoelectrons}

The change of the current, $I_{m}$, at the A-2 electrode was measured against a bias voltage in the range of $-11 \mathrm{~V}$ $+12 \mathrm{~V}$. For a positive bias voltage, the $I_{m}$ changed the polarity and the absolute current increased monotonously with the voltage. That means the photoelectrons, which would pass the electrode otherwise, are attracted with increasing the positive voltage. On the other hand, for a negative voltage, the current saturates at less than $5 \mathrm{eV}$. The saturation means that the photoelectrons are rejected by the negative potential. This indicates that the energy of photoelectrons is almost less than $5 \mathrm{eV}$. Here, we can define the current due to only the photoelectrons (photoelectron current, $\left.I_{p}\right)$ by $I_{p}=I_{m}(0 \mathrm{~V})-I_{m}(-11 \mathrm{~V})$,

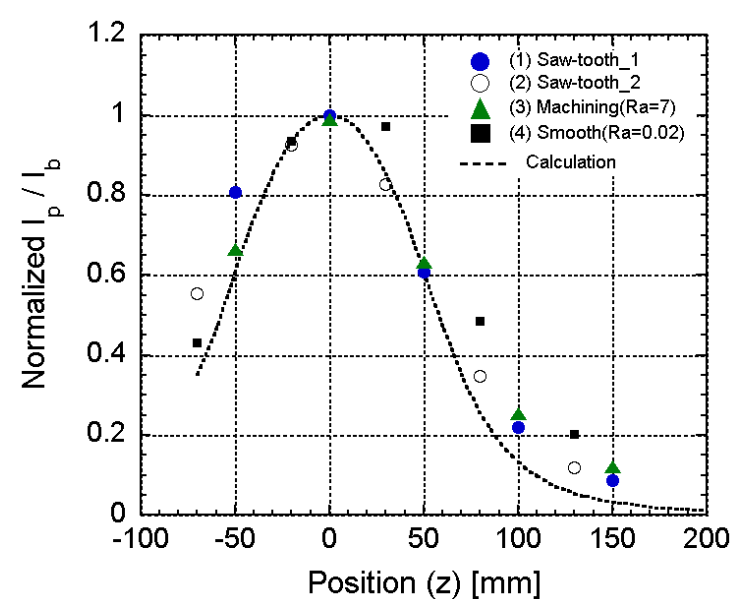

Figure 3: Axial distribution of $I_{p} / I_{b}$. The center of the irradiated area is $\mathrm{z}=0 \mathrm{~mm}$. The dotted line is the calculated value assuming the emission following the cosine law from the surface.

where $I_{m}(0 \mathrm{~V})$ and $I_{m}(-11 \mathrm{~V})$ are the measured current at a bias voltage of $0 \mathrm{~V}$ and $-11 \mathrm{~V}$, respectively.

\subsection{Photoelectron currents for four surfaces}

Figure 2 shows the measured $I_{p} / I_{b}$ (the photoelectron current normalized by the beam current) at each electrode (A-1 - A-5 and D) for four different surfaces above, (1) (4) in Sec.2. For the saw-tooth (1) and (2), the peak value is less than $6 \%$ of the smooth surface (4). The effectiveness of the saw-tooth surface to reduce the photoelectron yield was valid. Two saw-tooth surfaces, (1) and (2), have almost the same value but slightly smaller for the case (1). Even for the machined surface (3), the peak value is about $14 \%$ of the smooth surface (4). This means that a rough surface with a roughness of $\mathrm{Ra}=$ 7 serves as also a shallow but effective saw-tooth surface.

\subsection{Spatial distribution of the photoelectrons}

Figure 3 again shows the axial distribution of $I_{\mathrm{p}} / I_{\mathrm{b}}$ for four different surfaces, but the values are normalized by those near to the center of the irradiated area $(z=0)$. The dotted line is the calculated one assuming that the photoelectrons are emitted following the cosine law from only the directly irradiated area. Approximately the measured distribution indicates that the photoelectrons are emitted following the cosine law despite quite different surface structures. For saw-tooth (1) and (2), however, the backward values $(\mathrm{z}<0)$ are larger than in the case of (4) and also the calculated one. For the forward section $(z>0)$, all of the measured value are larger than the calculated ones. This may be due to the scattered photons in the chamber, especially from the electrode D.

The azimuthal distribution of $I_{p}$ was also measured and that for the case (4) was almost the same as the calculation using the cosine law. For the cases (1) - (3), especially for the case (2), the azimuthal distribution was flatter than the calculation. 
Table 1 Photoelectron yield, $\eta$. $\eta^{*}$ is the effective photoelectron yield considering the reflectivity, $R$, and given by $\eta /(1-R)$.

\begin{tabular}{c|ccc|ccc|ccc}
\hline Critical Energy & \multicolumn{3}{|c|}{$4.0 \mathrm{keV}$} & \multicolumn{3}{c|}{$195 \mathrm{eV}$} & \multicolumn{3}{c}{$45 \mathrm{keV}$} \\
Incident Angle & \multicolumn{3}{|c|}{$52 \mathrm{mrad}$} & \multicolumn{3}{c|}{$11 \mathrm{mrad}$} & \multicolumn{3}{c}{$11 \mathrm{mrad}$} \\
\hline & $\eta$ & $R[\%]$ & $\eta^{*}$ & $\eta$ & $R[\%]$ & $\eta^{*}$ & $\eta$ & $R[\%]$ & $\eta^{*}$ \\
\cline { 2 - 10 } Saw-tooth (1) & 0.016 & 0.18 & 0.016 & 0.052 & 1.2 & 0.052 & 0.053 & 1.8 & 0.053 \\
Machining (3) & 0.04 & 1.1 & 0.04 & & & & & & \\
Smooth (4) & 0.29 & 33.2 & 0.434 & 0.073 & 77 & 0.318 & 0.022 & 80.9 & 0.115 \\
\hline
\end{tabular}

\subsection{Photoelectron yield}

Assuming the cosine distribution of the emitted photoelectrons, the photoelectron yield can be estimated using the measured $I_{p}$ distribution. The results are summarized in Table.1. The $\eta$ represents the measured photoelectron yield calculated using the three values near $\mathrm{z}=0$ in Figure 3. The $\eta^{*}$ is the effective photoelectron yield corrected with the reflectivity, $R$, that is, $\eta^{*}$ is given by $\eta /(1-R)$. The reflection rate is very low for the surfaces (1) - (3). The $\eta^{*}$ for surfaces (1) and (3) are about $4 \%$ and $9 \%$ of case (4). For a reference, the results from CERN are also listed in the Table 1.

\subsection{Effect of positive potential}

The measurements so far have been performed in the condition that the electrode is set at the ground potential. Actually, however, the positron beam produces the positive potential around the beam and attracts the photoelectrons emitted from the surface. So the measurement was also performed applying $+70 \mathrm{~V}$ on the electrode. The obtained axial distributions of $I_{p} / I_{b}$ for the smooth and the saw-tooth surface are shown in Figure 4.

Compared to the case without bias voltage in Figure 2, it is found that the $I_{p} / I_{b}$ increased by one order of magnitude by applying $+70 \mathrm{~V}$ for both surfaces. The photoelectrons emitted from the surface were apparently attracted by the positive potential. However, it should be noted that the reduction of photoelectron yield (or more precisely, the photoelectron current measured at the beam position) by using the saw-tooth surface is still significant, less than $10 \%$ without considering the reflectivity.

\section{SUMMARY}

A saw-tooth surface was found to be effective to reduce the photoelectron yield, even for SR with a critical energy of $4.1 \mathrm{keV}$. The photoelectron yield for the saw-tooth surface was less than $6 \%$ of that for the smooth surface. A saw-tooth machined beam chamber seems to be a promising way to suppress the beam blow up observed in the KEKB LER. The reduction of the photoelectron seems valid even with a positive potential produced by the positron beam.

Note here that a difference in our experiments and the real ring is the existence of a bunched positron beam,

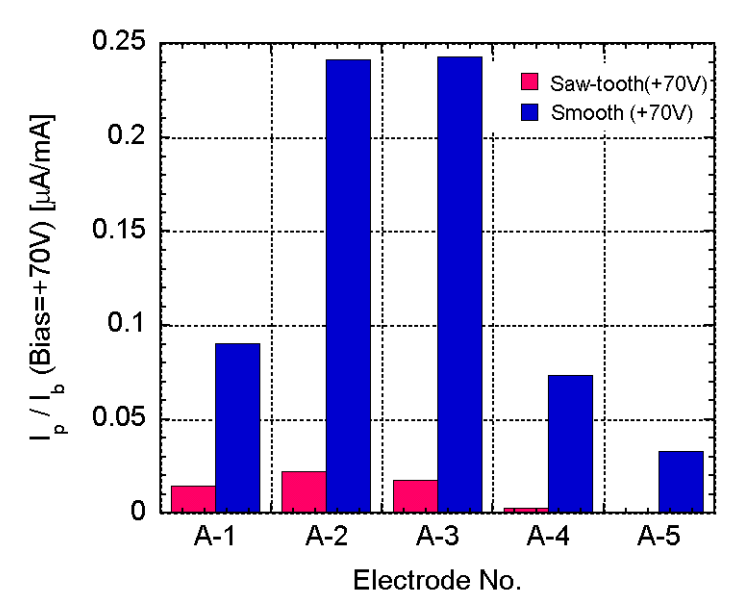

Figure 4: Axial distribution of $I_{p} / I_{b}$ with a bias voltage of $+70 \mathrm{~V}$ for the smooth surface and the saw-tooth surface.

where the multipactoring phenomena may occur [5]. The actual effect of a saw-tooth surface should be determined by a beam test using a test chamber installed in the ring. Another difference is that there are many scattered photons in the ring. The saw-tooth surface should be prepared at whole inner surface. Installation of a $2.6 \mathrm{~m}$ chamber with a rough surface is planned in the LER in this summer and the properties will be investigated.

\section{REFERENCES}

[1] S.Kurokawa, "Present Status of KEKB Project", 6th EPAC, Stockholm, June 1998.

[2] H.Hisamatsu et al., "Design of the vacuum system for KEKB", Vacuum, 47, 601,1996.

[3] H.Fukuma et al., "Observation of vertical beam blowup in KEKB Low Energy Ring", Proceedings of EPAC 2000, Vienna, p.1122.

[4] I.R.Collins and F.Zimmermann, "Electron Cloud Investigation, Electron Cloud Simulation", in LHC/SL Seminar, 11/11/99, http://wwwslap.cern.ch/collective.

[5] Y.Suetsugu, "OBSERVATION AND SIMULATION OF THE NONLINEAR DEPENDENCE OF VACUUM PRESSURES ON THE POSITRON BEAM CURRENT AT THE KEKB", this proceedings. 